\title{
Evaluación de las propiedades psicométricas del Brief COPE, su relación con el NEO PI-R y diferencias de género en Brasil*
}

\author{
Evaluation of Psychometric Properties of the Brief COPE, its \\ Relationship with the NEO PI-R and Gender Differences in Brazil
}

Recibido: noviembre 16 de 2012 | Revisado: noviembre 19 de 2013 | Aceptado: abril 16 de 2014

\author{
CONSUELO MORÁN ** \\ Universidad de León, España \\ LUZ MARINA MENDEZ HiNOJOSA *** \\ Mónica Teresa GONZÁlez RAMÍREZ \\ RENÉ LANDERO-HERNÁNDEZ \\ Universidad Autónoma de Nuevo León, México
}

ESTER MENEZES

Uninorte Laureate Internacional Universities, Brasil

doi:10.11144/Javeriana.UPSY13-4.eppb

Para citar este artículo: Morán, C., Méndez, L. M., González, M. T., Landero-Hernández, R., \& Menezes, E. (2014). Evaluación de las propiedades psicométricas del Brief COPE, su relación con el NEO PI-R y diferencias de género en Brasil. Universitas Psychologica, 13(4), 1305-1320. http://dx.doi. org/10.11144/Javeriana.UPSY13-4.eppb

* Este trabajo es producto de la tesis doctoral de Ester Menezes dos Anjos. Agradecemos a Uninorte Laureate Internacional Universities de Manaos (Brasil) por facilitar su realización.

** Correo electrónico: consuelo.moran@unileon.es

**** Facultad de Psicología. Av. Universidad s/n Ciudad Universitaria San Nicolás de los Garza Nuevo León C. P. 66451, México. Tel. (81) 83294050. Correos electrónicos: lucymendezhinojosa@hotmail.com; monygzz77@yahoo.com

\section{RESUMEN}

El NEO PI-R es una escala base para examinar la personalidad y el Brief COPE es un instrumento, poco difundido en Brasil, para examinar el afrontamiento. Por ello, el presente estudio transversal con muestra brasileña tiene como objetivos evaluar las propiedades psicométricas del Brief COPE, examinar su relación con el NEO PI-R, así como también evaluar las diferencias de género en el NEO PI-R y el Brief Cope. Participaron 899 adultos. El análisis factorial no confirmó la estructura original del Brief COPE. El análisis factorial exploratorio evidenció una solución de ocho factores, que fue corroborada por el análisis factorial confirmatorio. Se encontraron correlaciones entre las cinco dimensiones de la personalidad y los factores del afrontamiento, así como también diferencias significativas de género. Palabras clave personalidad; afrontamiento; Big Five

\section{A B S T R A C T}

The NEO PI-R is a major scale to examine the personality, and the Brief COPE is a little known instrument in Brazil to examine the coping. As this cross-sectional study with Brazilian sample aims to: evaluate the psychometric properties of the Brief COPE; examine its relationship with the NEO PI-R, and evaluate gender differences in the NEO PI-R and Brief Cope. The participants were 899 adults. The factor analysis did not confirm the original structure of the Brief COPE. The exploratory factorial analysis showed an eight factor solution, which was confirmed by the confirmatory factorial analysis. Correlation was found between the five dimensions of personality and coping factors, as well significant differences between genders.

Keywords

Personality; coping; Big Five 


\section{Introducción}

En las últimas décadas, la psicología de la personalidad ha hecho grandes avances identificando los rasgos más consistentes de las individualidades psicológicas (McAdams \& Olson, 2010). Está suficientemente aclarado que los rasgos de la personalidad son robustos predictores de la conducta, y que esta predicción se mantiene a lo largo del tiempo y para la mayoría de las situaciones. De igual forma, la personalidad predice importantes resultados de la vida como la calidad de las relaciones interpersonales, la adaptación a los cambios, el éxito en el trabajo, la felicidad, la salud e incluso la exposición a riesgos mortales (Lodi-Smith \& Roberts, 2012).

La personalidad constituye también uno de los recursos de la persona que facilita o dificulta el proceso de afrontamiento del estrés, ya que puede intervenir directamente en la selección de la estrategia, facilitando o restringiendo el uso de estrategias específicas o, indirectamente, influyendo en la naturaleza y severidad del estrés experimentado o en la efectividad de las estrategias elegidas (Bolger \& Zuckerman, 1995).

Un modelo de la personalidad que ha mostrado ser particularmente útil para comprender el afrontamiento es el de los Cinco Factores (Big Five). Se trata de una taxonomía de amplia base de las dimensiones que bien pueden representar el número mínimo de rasgos necesarios para describir la personalidad (Costa \& McCrae, 1985). Un importante corpus de literatura científica apoya la validez de constructo de esta conocida taxonomía (McCrae $\&$ Costa, 2008). El modelo de los cinco factores es una organización jerárquica de los rasgos de la personalidad en términos de cinco dimensiones básicas (o factores) denominadas: Neuroticismo, Extraversión, Apertura a la Experiencia, Amabilidad y Responsabilidad, que a su vez se subdividen en facetas.

Algunos investigadores estiman que la personalidad también puede predecir el afrontamiento porque sistemáticamente influye en las valoraciones, metas, expectativas y procesos cognitivos de las situaciones estresantes (Gunzerath, Connelly, Albert, \& Knebel, 2001; Watson \& Hubbard, 1996).
Por tanto, aunque ciertos autores consideren que la presencia de determinados rasgos de personalidad aumenta la vulnerabilidad a los procesos relacionados con el estrés (Morán, 2006), para otros, la relación entre personalidad y estrés no es directa, es decir, las disposiciones de personalidad no aumentan la vulnerabilidad al estrés, sino que generan en las personas una forma ineficaz de afrontar los estresores situacionales y en consecuencia un incremento del estrés.

Respecto al proceso de afrontamiento, la teoría de Lazarus y Folkman (1984) es la que ha dominado en dicho campo durante los últimos 20 años (Shiloh \& Orgler-Shoob, 2006). En esta teoría, se define el afrontamiento como los intentos cognitivos, emocionales y conductuales para manejar demandas que son evaluadas como exigentes o que exceden la propia capacidad del individuo para adaptarse. El afrontamiento es cualquier esfuerzo para manejar el estrés, y se evidencia su ejecución mediante las acciones de las personas para evitar ser dañadas por las adversidades de la vida. Estas acciones han sido conceptualizadas como estrategias de afrontamiento.

Las estrategias de afrontamiento son acciones concretas para modificar las condiciones del estresor, mientras que los recursos son características personales y sociales que posee la persona para hacer frente a los estresores (Sandín, 2003).

Respecto a lo anterior, se identifican los dos constructos de interés en el presente estudio: la personalidad y el afrontamiento. La confluencia de dichas variables la sintetiza Bolger (1990, p. $525)$ quien define al afrontamiento como "la personalidad en acción bajo estrés" y Vollrath (2001) quien ha sugerido que el afrontamiento debería ser redefinido como un proceso de la personalidad.

La teoría de los Cinco Grandes (Big Five) considera que la personalidad está conformada por cinco grandes factores o dimensiones (Costa \& McCrae, 1985) que podrían ser definidos como sigue.

1. Neuroticismo. Es el factor que indica emocionalidad negativa, hiperreactividad fisiológica al estrés y conductas de inhibición de la acción (McCrae \& John, 1992). Ya que el neuroticismo implica intensas respuestas emocionales y físicas al 
estrés, debería estar ligado al intento de minimizar la estimulación desagradable a través de estrategias como evitación, desconexión, uso de sustancias o desahogo emocional. En general, los estudios han mostrado que los sujetos altos en neuroticismo usan un afrontamiento pobre ya que eligen estrategias ineficientes que pueden servir incluso para exacerbar las situaciones estresantes. En el estudio de O'Brien y DeLongis (1996) se examinaron las estrategias con un amplio número de estresores, encontrando que los más altos en neuroticismo informaban de inferiores niveles de solución de problemas, así como de la presencia de acciones de evitación-huida (escape) y de autoinculpación. No obstante lo anterior, los altos en neuroticismo no necesariamente adoptan estrategias rígidas de afrontamiento, como tampoco carecen de la capacidad de afrontar las situaciones utilizando estrategias adaptativas. Lo que ocurre es que eligen estrategias equivocadas, dada la situación particular a la que se enfrentan. Según DeLongis y Holtzman (2005), el patrón general encontrado en los estudios sugiere que sus altos niveles de emociones negativas impiden a los altos en neuroticismo servirse de su capacidad para elegir estrategias de afrontamiento apropiadas.

2. Extraversión. Comprende emocionalidad positiva, sociabilidad, asertividad, niveles altos de actividad y consideración hacia los demás (McCrae \& John, 1992). En general, los resultados de las investigaciones sobre afrontamiento indican que los individuos más altos en extraversión son más activos y eficientes en el afrontamiento, teniendo la energía y el optimismo requerido para iniciar y persistir en el esfuerzo del afrontamiento (Vollrath, 2001). Es más probable que los altos en extraversión utilicen modos de afrontamiento más variados y a que lo hagan de forma más efectiva, al destacar en reevaluación cognitiva, solución activa de problemas y búsqueda de apoyo social, siendo menos probable que utilicen estrategias centradas en la emoción como evitación o afrontamiento religioso.

3. Apertura a la experiencia. También denominada apertura mental o simplemente apertura, indica integración activa, sensibilidad estética, atención a los sentimientos interiores, preferencia por la variedad, curiosidad intelectual e independencia de juicio. Los sujetos altos en apertura deberían utilizar más planificación, reinterpretación cognitiva, aceptación y distracción, ya que apertura requiere la habilidad para considerar nuevas perspectivas. Los hallazgos de DeLongis y Holtzman (2005) indican que los altos en apertura muestran niveles inferiores de reevaluación cognitiva. Existen diferencias con los hallazgos de McCrae y Costa (2008), sin que esté claro si son debidas a especificidad situacional en los efectos de la personalidad sobre el afrontamiento, o bien a diferencias metodológicas entre los estudios. Además, la reinterpretación cognitiva varía según los distintos tipos de estresores, lo mismo que también el nivel de apertura interactúa con el tipo de estresor al predecir la utilización del afrontamiento. De los individuos altos en apertura se espera que en el afrontamiento sean adaptativos, flexibles y preocupados por los otros y por el mundo y su entorno, como así se han mostrado al responder más empáticamente en situaciones estresantes que implican a personas íntimas en cuanto comparados a cuando se enfrentan a estresores no interpersonales (O’Brien \& DeLongis, 1996).

4. Amabilidad. Significa ser confiado, altruista, complaciente y compasivo (McCrae \& John, 1992). No es sorprendente que amabilidad sea la dimensión de la personalidad más fuertemente asociada con modos de afrontamiento de apoyo social, dado que amabilidad representa la principal dimensión de las relaciones interpersonales. Así, se ha hallado que los altos en amabilidad informan de menor afrontamiento de confrontación y de mayor búsqueda de apoyo que los bajos en amabilidad. También se encontró que los altos en amabilidad variaban la utilización de respuesta empática y de confrontación según fueran los estresores. Todavía más interesante es la falta de evidencia de que amabilidad esté relacionada con respuestas de afrontamiento, lo que sugiere que el rol de amabilidad en el afrontamiento puede estar limitado precisamente a estresores interpersonales (DeLongis \& Holtzman, 2005).

5. Responsabilidad. Representa orden, meticulosidad, planificación de metas, persistencia en el esfuerzo, motivación por el logro. Responsabilidad 
debería predecir estrategias enfocadas a la solución del problema y la regulación de la emoción, lo que requiere planificación y persistencia para encarar las dificultades y control de las estrategias como la distracción y la reinterpretación cognitiva así como el alejamiento de la atención de los pensamientos negativos hacia actividades y pensamientos positivos. Los altos en responsabilidad informan que reaccionan de forma más empática que los bajos en responsabilidad (DeLongis \& Holtzman, 2005). Responsabilidad interactúa con el tipo de estresor en la predicción de autoinculpación, también podría predecir bajos niveles de desconexión, uso de sustancias y afrontamiento centrado en la emoción (Vollrath, 2001).

Por su parte, las estrategias de afrontamiento han sido categorizadas por Carver (1997) en su instrumento Brief COPE, siendo Morán, Landero y González (2010) quienes las han definido de la siguiente forma:

1. Afrontamiento activo. Implica iniciar acciones directas e incrementar los propios esfuerzos para eliminar o reducir al estresor, en un intento de solucionar el problema de manera sensata.

2. Planificación. Consiste en pensar acerca de cómo afrontar al estresor, planificar estrategias de acción, identificar los pasos a dar y la dirección de los esfuerzos a realizar.

3. Apoyo instrumental. Implica procurar ayuda, consejo, y solicitar información a personas que son competentes acerca de lo que se debe hacer.

4. Uso de apoyo emocional. Es el hecho de conseguir apoyo emocional, simpatía y comprensión de otros.

5. Autodistracción. Significa concentrarse en otros proyectos, intentando distraerse con otras actividades, para tratar de no concentrarse en el estresor.

6. Desahogo. Es la tendencia a expresar o descargar los sentimientos que produce el estresor, lo que lleva al aumento de la conciencia del propio malestar emocional.

7. Desconexión conductual. Es reducir los esfuerzos para tratar con el estresor, incluso renunciando al esfuerzo para lograr las metas con las cuales se interfiere al estresor.
8. Reinterpretación positiva. Consiste en buscar el lado positivo y favorable del problema e intentar mejorar o crecer a partir de la situación.

9. Negación. Implica negar la realidad del suceso estresante.

10. Aceptación. Es el hecho de aceptar lo que está ocurriendo.

11. Religión. Es la tendencia a volver hacia la religión en momentos de estrés, aumentado la participación en actividades religiosas.

12. Uso de sustancias (alcohol, medicamentos). Implica tomar alcohol o consumir otras sustancias con el fin sentirse bien o para ayudarse a soportar al estresor.

13. Humor. Consiste en hacer bromas sobre el estresor o reírse de las situaciones estresantes, haciendo burlas de la misma.

14. Autoinculpación. Lleva a criticarse y culpabilizarse por lo sucedido.

El interés por estudiar el afrontamiento puede hallarse en la creencia de que en una cierta cultura los modos de afrontamiento serán más o menos efectivos para promover el bienestar emocional y orientar problemas causantes de estrés, al mismo tiempo que se pueda usar esa información para diseñar intervenciones para ayudar a la gente a enfrentarse más eficientemente al estrés de sus vidas. Sin embargo, aún no se ha determinado la efectividad del afrontamiento, permaneciendo como una de las cuestiones más desconcertantes en la investigación sobre del mismo.

Somerfield y McCrae (2000) afirman que los investigadores del estrés y el afrontamiento se han distanciado de la psicología clínica, cuando se han definido a sí mismos como estudiosos de un campo que abarca procesos psicológicamente normales. Así, el afrontamiento se considera una respuesta racional a un problema objetivo, distinto de los procesos psicopatológicos y desadaptativos. Según los autores, se ha de reconceptualizar el estrés y el afrontamiento como una parte integral del espectro de la adaptación, ya que las diferencias entre el afrontamiento inefectivo y el mal ajuste psicológico son más cuantitativas que cualitativas. Así, cuando se refieren a expectativas más realistas respecto a las contribuciones del afrontamiento, afirman: "Es una 
cuestión crítica en la historia actual de la investigación sobre los procesos adaptativos el considerar muy cuidadosamente la contribución potencial de la investigación sobre estrés y afrontamiento a la mejora de la condición humana" (Somerfield \& McCrae, 2000, p. 623).

En suma, la evidencia del afrontamiento examinado a través de muy diversas situaciones estresantes sugiere que los Cinco Factores (Big Five) de la personalidad interactúan con el tipo de estresor para desencadenar respuestas de afrontamiento, también que la gente varía su afrontamiento a través de las situaciones para satisfacer las necesidades de la situación. Algunos (e. g., los altos en apertura) pueden variar su conducta más adaptativamente que otros (e. g., los altos en neuroticismo). No obstante, parece que se necesita examinar el afrontamiento tanto a través de las situaciones como en las personas, para incrementar la comprensión del rol de la personalidad en el estrés, el afrontamiento y la adaptación (DeLongis \& Holtzman, 2005).

La mayoría de las investigaciones sobre la relación de la personalidad con el afrontamiento se han venido realizando con la versión abreviada del NEO PI-R, como es el inventario NEO-FFI de 60 ítems, de Costa y McCrae (1992). Son menos las investigaciones realizadas con el inventario completo, siendo aún más escasos los estudios que relacionan las facetas con las estrategias de afrontamiento. También la mayor parte de estos estudios se han centrado en el papel del neuroticismo, la extraversión y la apertura a la experiencia (Connor-Smith \& Flachsbart, 2007). Las futuras investigaciones deberían enfocarse a todos los factores y a las facetas que más claramente influyen en el afrontamiento, lo que ayudaría significativamente a la comprensión del proceso del estrés y de su afrontamiento, según sugieren posteriormente Carver y Connor-Smith (2010).

Numerosos instrumentos han sido utilizados para medir y clasificar las estrategias de afrontamiento. Así, el Ways of Coping Checklist de Folkman y Lazarus (1988) reveló la existencia de ocho estrategias clasificadas en dos dimensiones: afrontamiento dirigido al problema y afrontamiento dirigido a la emoción. Siguiendo ese modelo teórico,
Carver, Scheier y Weintraub (1989) desarrollaron el Coping Orientation to Problems Experienced (COPE), del que más adelante Carver (1997) presentó una versión abreviada de 28 ítems, el Brief COPE, que mide 14 estrategias de afrontar los problemas con estrés. Una versión en español del Brief COPE fue realizada por Morán et al. (2010).

No obstante los grandes esfuerzos realizados por los investigadores en torno a la creación de medidas efectivas para evaluar el afrontamiento, hay escasez de estudios que validen instrumentos de medición para la población brasileña. Además, se ha detectado, en la misma población, la necesidad de replicar estudios sobre la relación de la personalidad y el afrontamiento ya realizados en otros contextos socioculturales. Por esta razón, en este trabajo, se plantean los siguientes objetivos para una muestra brasileña: 1) evaluar las propiedades psicométricas Brief COPE, 2) examinar la relación entre las subescalas del NEO PI-R y el Brief COPE y 3) evaluar las diferencias de género en el NEO PI-R y el Brief Cope.

\section{Método}

\section{Participantes}

Los participantes fueron 899 adultos brasileños, estudiantes en dos universidades privadas de Manaos (Brasil). El rango de edad estaba entre 17 y 60 años con media de 28.39 años, desviación estándar de 7.52 y el $64 \%$ eran mujeres. Es preciso destacar que debido a que el $76.5 \%$ de la muestra trabajaba durante el día y estudiaba en horario nocturno, es probable la presencia en los participantes de diversas situaciones de estrés ante las cuales se les requiere llevar a cabo estrategias de afrontamiento.

\section{Instrumentos de medida}

Inventario de Personalidad NEO Revisado (NEO PI-R)

Es la versión brasileña del NEO PI-R de Costa y McCrae (1992). Es un cuestionario de 240 ítems diseñado para operacionalizar el modelo de los Cin- 
co Factores de personalidad. Pretende medir esos cinco factores, que a su vez contienen seis facetas cada uno. El NEO PI-R, en cuanto a sus factores lo mismo que en cuanto a sus facetas componentes, posee propiedades psicométricas adecuadas (Costa, McCrae, \& Dye, 1991). Para este estudio, la fiabilidad por alfa de Cronbach en los Cinco Factores fue de 0.87 en Neuroticismo (N), 0.83 en Extraversión (E), 0.74 en Apertura a la experiencia (O), 0.76 para Amabilidad (A) y de 0.86 en Responsabilidad (C).

\section{Brief COPE}

Fue diseñado por Carver (1997) para medir el afrontamiento de los problemas con estrés, y en su versión original indica adecuada confiabilidad y validez. En este estudio, se aplicó la versión portuguesa de Pais-Ribeiro y Rodrigues (2004), traducida y adaptada al portugués, reportando una adecuada confiabilidad e indicando que dicha versión conserva las propiedades psicométricas de la original. Este cuestionario de 28 ítems ( 2 ítems por escala) evalúa las 14 estrategias de afrontamiento para las que los autores reportan los siguientes índices de fiabilidad (alfa de Cronbach): Afrontamiento activo ( $\alpha=$ 0.65), Planificación ( $\alpha=0.7)$, Búsqueda de apoyo emocional ( $\alpha=0.79$ ), Búsqueda de apoyo social ( $\alpha$ $=0.81)$, Religión $(\alpha=0.8)$, Revaluación positiva ( $\alpha=0.74)$, Aceptación $(\alpha=0.55)$, Negación ( $\alpha$ $=0.72)$, Humor $(\alpha=0.83)$, Autodistracción $(\alpha=$ 0.67), Autoinculpación ( $\alpha=0.62)$, Desconexión ( $\alpha$ $=0.78)$, Desahogo $(\alpha=0.84)$ y Uso de sustancias $(\alpha=0.81)$.

\section{Procedimiento}

Los cuestionarios fueron administrados por psicólogas entrenadas para realizar este estudio. Se aplicaron en el contexto del aula, donde los grupos tenían entre 30 y 60 alumnos. La recogida de datos se realizó entre junio y noviembre de 2009. De los 955 cuestionarios entregados todos fueron recogidos, aunque se desecharon 56 por contar con más del $5 \%$ de valores perdidos de acuerdo con el criterio presentado por Hernández-Sampieri, Fernández-Collado y Baptista-Lucio (2010).

\section{Procedimiento de análisis estadístico}

En un primer momento se evaluaron las propiedades psicométricas del Brief COPE, iniciando con la fiabilidad de sus 14 escalas. En cuanto a la validez del instrumento, se aplicó la prueba de esfericidad de Barlett $(1950,1951)$, para asegurarse de que la matriz de correlaciones de cada subescala fuera significativa $(p<0.05)$ y poder rechazar la hipótesis de independencia de las variables.

Como parte de la evaluación de las propiedades psicométricas del Brief COPE, se exploró su estructura y su dimensionalidad mediante el análisis factorial exploratorio (AFE). Se aplicó como método de extracción el análisis de los componentes principales (PCA), que es el método utilizado más frecuentemente y el apropiado cuando la meta es la reducción de datos (Fabrigar, MacCallum, Wegener, \& Strahan, 1999); del mismo modo, ha sido diseñado con el propósito de servir en una primera fase exploratoria al análisis factorial (Landero \& González, 2006). Debido a que el Brief COPE es un inventario de 28 ítems agrupados en 14 escalas, se buscó mediante la rotación VARIMAX incrementar la independencia de sus factores para poder comprobar su estructura.

Posteriormente, a través del coeficiente alfa de Cronbach, se estimó la confiabilidad del instrumento para cada factor. Se corroboró la estructura factorial propuesta por Carver (1997) y la obtenida en el AFE mediante el análisis factorial confirmatorio (AFC).

Los estadísticos de bondad de ajuste que se consideraron en el AFC fueron los siguientes: razón de chi-cuadrado entre los grados de libertad $(\chi 2 / g l)$ que se utiliza para comparar la magnitud de $\chi 2$ respecto a los grados de libertad, para un buen ajuste esta razón deber ser tan pequeña como sea posible, los valores menores a 3 demuestran ajuste bueno o aceptable (Schermelleh-Engel, Moosbrugger, y Müller, 2003); GFI (goodness of fit index) que es un índice desarrollado por Jöreskog y Sörbom (1984), sus valores son menores o iguales a 1, y un valor de 1 indica un ajuste perfecto; AGFI (adjusted goodness of fit index) fue desarrollado por los mismos autores y corrige el estadístico GFI por los 
grados de libertad y el número de variables, tanto GFI como AGFI alcanzan el valor de 1 cuando todos los residuos son nulos (Ruíz, 2000); RMSEA (root mean square error of approximation), donde valores de 0.05 o menos revelan un buen ajuste del modelo, considerando sus grados de libertad, valores de 0.05 a 0.08 o más, podrían indicar un error razonable de aproximación y valores mayores a 0.1 dicen que el modelo no es adecuado (Browne \& Cudeck, 1993).

Como consecuencia de la estrecha relación de la personalidad y el afrontamiento se examinó la relación del Brief COPE y el NEO PI-R. A continuación, se obtuvieron estadísticos descriptivos agrupando por género las puntuaciones obtenidas en ambas subescalas y, para analizar si las diferencias por género eran significativas, se obtuvo el estadígrafo $t$ de Student para cada pareja de medias (varón-mujer en cada subescala).

\section{Resultados}

Respecto a la evaluación de las propiedades psicométricas del Brief COPE, se encontró en un primer momento que la consistencia interna era baja en algunas escalas, variando desde 0.4 en Autoinculpación a 0.72 en Desahogo. Se estimó la medida de adecuación muestral Kaiser-Meyer-Olkin (KMO), cuyo valor fue de 0.788 ; dado que para ser aceptable el índice KMO debe ser mayor a 0.5, el resultado obtenido indicó adecuación muestral y viabilidad del análisis factorial. La prueba esfericidad de Barlett fue significativa $\left(\chi^{2}=5272.869 ; g l=378 ; p\right.$ $=0.01)$, lo que llevó al rechazo de la hipótesis de independencia de las variables y dedujo que era adecuado continuar con el análisis factorial. En síntesis, ambos valores, $\mathrm{KMO}$ y Barlett, justificaron plenamente la aplicación del análisis factorial exploratorio.

El presente estudio tomó como base la agrupación factorial inicial del Brief COPE presentada por Carver (1997); de esta manera, para la evaluación de las soluciones obtenidas en el AFE se analizaron las saturaciones de los ítems en los factores partiendo de 14 factores, siendo el criterio de inclusión del ítem al factor 0.4 (Hair, Anderson, Tatham, \& Black, 1999).

Tomando como base las cargas factoriales, así como su mejor agrupación para una interpretación lógica, se obtuvo en una solución de 8 factores (Tabla 1). Los ítems del Brief COPE, excepto el ítem 5 perteneciente a la subescala de Negación, alcanzaron cargas superiores a 0.4. La carga del ítem 5 fue de 0.363 , por lo que se eliminó de la escala.

A partir del AFE se buscó mejorar la consistencia interna y utilizar un menor número de factores. Por el criterio de Kaiser (autovalores de la matriz de correlaciones mayores a 1), se definieron 8 factores que explicaron el $55.22 \%$ de la varianza total. Tras la rotación VARIMAX, el primer factor quedó definido por seis indicadores (ítems 2, 6, 10, 14, 18 y 26) con consistencia interna alta $(\alpha=0.71)$ y fue denominado Afrontamiento centrado en la solución; el segundo agrupó 4 indicadores (ítems 8, 11, 25 y 27) con consistencia interna baja $(\alpha=0.55)$ y fue denominado Desconexión-Autoinculpación; el tercero quedó formado por 4 indicadores (ítems 1 , 9,17 y 28) con consistencia interna alta $(\alpha=0.73)$ y se denominó Apoyo socioemocional; el cuarto quedó integrado por dos indicadores (ítems $16 \mathrm{y}$ 20) con consistencia interna alta $(\alpha=0.72)$ y se denominó Religión; el quinto quedó conformado por los dos indicadores (ítems 15 y 24) con consistencia interna adecuada $(\alpha=0.6)$ y se denominó Uso de sustancias; el sexto quedó definido por dos indicadores (ítems 7 y 19) con consistencia interna baja $(\alpha=0.54)$ y se denominó Humor; el séptimo quedó configurado por dos indicadores (ítems 3 y 21) con consistencia interna baja $(\alpha=0.4)$ y se denominó Aceptación y el octavo quedó conformado por 5 indicadores (ítems 4, 12, 13, 22 y 23) con consistencia interna adecuada $(\alpha=0.64)$ y se denominó Evasión (Tabla 1). Es importante aclarar que el valor del coeficiente alfa se ve afectado por el número de ítems, siendo dos ítems una cantidad muy reducida para lograr valores de consistencia interna adecuados.

En el AFC se buscó contrastar la estructura propuesta por Carver (1997) y se respetaron los 14 factores. Sin embargo, en un primer modelo, fue preciso establecer tres factores de orden superior 
TABLA 1

Matriz de componentes rotados

\begin{tabular}{|c|c|c|c|c|c|c|c|c|c|}
\hline \multirow{2}{*}{ Ítem } & \multirow{2}{*}{ Escala } & \multicolumn{8}{|c|}{ Saturaciones en el factor } \\
\hline & & 1 & 2 & 3 & 4 & 5 & 6 & 7 & 8 \\
\hline 26 & Planificación & 0.693 & & & & & & & \\
\hline 6 & Planificación & 0.658 & & & & & & & \\
\hline 14 & Reinterpretación positiva & 0.578 & & & & & & & \\
\hline 2 & Afrontamiento activo & 0.563 & & & & & & & \\
\hline 10 & Afrontamiento activo & 0.557 & & & & & & & \\
\hline 18 & Reinterpretación positiva & 0.528 & & & & & & & \\
\hline 9 & Apoyo emocional & & 0.744 & & & & & & \\
\hline 1 & Apoyo social & & 0.742 & & & & & & \\
\hline 28 & Apoyo social & & 0.713 & & & & & & \\
\hline 17 & Apoyo emocional & & 0.641 & & & & & & \\
\hline 23 & Desahogo & & & 0.726 & & & & & \\
\hline 12 & Desahogo & & & 0.710 & & & & & \\
\hline 13 & Negación & & & 0.602 & & & & & \\
\hline 4 & Autodistracción & & & 0.514 & & & & & \\
\hline 22 & Autodistracción & & & 0.463 & & & & & \\
\hline 25 & Desconexión & & & & 0.636 & & & & \\
\hline 27 & Autoinculpación & & & & 0.605 & & & & \\
\hline 11 & Desconexión & & & & 0.567 & & & & \\
\hline 8 & Autoinculpación & & & & 0.540 & & & & \\
\hline 19 & Humor & & & & & 0.751 & & & \\
\hline 7 & Humor & & & & & 0.670 & & & \\
\hline 20 & Religión & & & & & & 0.823 & & \\
\hline 16 & Religión & & & & & & 0.813 & & \\
\hline 15 & Uso de sustancias & & & & & & & 0.821 & \\
\hline 24 & Uso de sustancias & & & & & & & 0.793 & \\
\hline 3 & Aceptación & & & & & & & & 0.798 \\
\hline 21 & Aceptación & & & & & & & & 0.489 \\
\hline
\end{tabular}

Fuente: elaboración propia

relacionados con las estrategias de afrontamiento: Centrado en la emoción o Pasivo; Centrado en el problema o Activo y Estrategias inusuales.

En este primer modelo (véase Figura 1), la problemática en la dimensión Estrategias inusuales la suscitó el factor Humor ya que al fijar a la unidad dos parámetros de este factor de orden superior (ítems 7 y 19), el programa AMOS sí procedía con el cálculo; no obstante, la solución resultó inadmisible al ser el coeficiente $\beta$ del factor Planificación mayor a $1(\beta=1.01)$ y la varianza de su residuo negativa $\left(S^{2}{ }_{\text {e } 33]}=-0.002\right)$.

En un segundo modelo, se consideró al factor Humor dentro de la dimensión de afrontamiento
Centrado en la emoción o pasivo, por lo que se pudo realizar el cálculo sin necesidad de fijar a la unidad un segundo parámetro por problemas de indefinición, pero nuevamente la solución resultó inadmisible. El coeficiente $\beta$ de un indicador del factor Uso de sustancias (ítem 24) fue mayor de 1 $(\beta=1.04)$ y la varianza del residuo fue negativa $\left(S^{2}{ }_{[\mathrm{e} 11]}=-0.012\right)$.

Debe señalarse que la correlación entre la dimensión de Centrado en la emoción y Estrategias inusuales fue muy alta $(r=0.97)$ en el primer modelo y alta en el segundo $(r=0.79)$. Por lo tanto, se unieron ambos factores en uno y así se creó un tercer modelo. En ese tercer modelo no hubo 
Figura 1. Modelo estandarizado de 14 factores de orden inferior con dos indicadores cada uno y tres factores de orden superior correlacionados estimado por GLS (humor determinado con afrontamiento inusual).

Fuente: elaboración propia

problemas de indefinición y la solución fue admisible. Todos los parámetros fueron significativos y la correlación entre ambos factores resultó alta $(r$ $=0.72$ ), con un $52 \%$ de varianza compartida. Se introdujo una correlación entre los residuos de dos indicadores (ítems 19 y 24), resultando los valores de los índices de ajuste considerados de buenos $($ AGFI $=0.91$, RMSEA $=0.046)$ a adecuados $\left(\chi^{2}\right)$ $g l=2.91, \mathrm{GFI}=0.92)$, sin embargo, la prueba chicuadrado rechazó la hipótesis nula de bondad de ajuste $\left(\chi^{2}[334, N=899]=971.71, p<0.01\right)$.

Por lo que se procedió a contrastar la estructura factorial obtenida en el AFE con un modelo de 8 factores correlacionados. Inicialmente, se obtuvo una solución inadmisible al ser el coeficiente $\beta$ del ítem 19 (indicador del factor Humor) mayor a la unidad y la varianza de su residuo negativa. Se eliminaron 8 correlaciones no significativas y persistió el problema. Tras revisar la tabla de índices de modificación se introdujo una determinación del ítem 7, que es el otro indicador del factor Humor, con el factor Desconexión-Inculpación, con lo que se obtuvo una solución admisible. Todos los parámetros fueron significativos y los índices de ajuste fueron de buenos $(\mathrm{AGFI}=0.92, \mathrm{RMSEA}=0.042$ ) a adecuados $\left(\chi^{2} / g l=2.63, \mathrm{GFI}=0.93\right)$, aunque por la prueba chi-cuadrado se rechazó la hipótesis nula de bondad de ajuste $\left(\chi^{2}[307, N=899]=822.14, p\right.$ $<0.01)$. Por la prueba de la diferencia de los estadísticos chi-cuadrado, el ajuste de este modelo de 8 factores correlacionados es mejor que el modelo jerarquizado anterior $\left(d \chi^{2}[27]=149.57, p<0.01\right)$ (Figura 2).

Ahora bien, se establecieron las correlaciones entre los factores del Brief COPE y las escalas del NEO PI-R y, como se muestra a continuación en la Tabla 2, se presentaron correlaciones significativas en el $75 \%$ de las relaciones. Las correlaciones 
FIGURA 2. Modelo estandarizado de 8 factores correlacionados.

Fuente: elaboración propia

más fuertes se encontraron en Responsabilidad con Afrontamiento Centrado en la Solución $(r=$
0.389) y en Neuroticismo con Desconexión-Autoinculpación $(r=0.421)$ y con Evasión $(r=0.432)$.

\section{TABLA 2}

Correlaciones Brief COPE y NEO PI-R

\begin{tabular}{lccccc} 
& Neuroticismo & Extraversión & Apertura & Amabilidad & Responsabilidad \\
\cline { 2 - 5 } Afrontamiento centrado en la solución & $-0.258^{* *}$ & $0.337^{* *}$ & $0.249 * *$ & $0.206^{* *}$ & $0.389^{* *}$ \\
Desconexión-Autoinculpación & $0.421^{* *}$ & $-0.194^{* *}$ & $-0.089^{* *}$ & $-0.1 * *$ & $-0.336^{* *}$ \\
Apoyo socioemocional & $0.171^{* *}$ & $0.209^{* *}$ & 0.024 & $0.158^{* *}$ & 0.016 \\
Evasión & $0.432^{* *}$ & -0.023 & $-0.072 *$ & $-0.218^{* *}$ & $-0.201^{* *}$ \\
Religión & $-0.068^{*}$ & $0.17^{* *}$ & 0.051 & $0.245^{* *}$ & $0.153^{* *}$ \\
Uso de sustancias & $0.111^{* *}$ & -0.033 & 0.011 & $-0.093^{* *}$ & $-0.069^{*}$ \\
Humor & -0.055 & $0.22 * *$ & $0.095^{* *}$ & 0.004 & -0.026 \\
Aceptación & $-0.103^{* *}$ & $0.138^{* *}$ & 0.059 & $0.173 * *$ & $0.11^{* *}$ \\
\hline
\end{tabular}

* La correlación es significativa al nivel 0.05 (bilateral).

** La correlación es significativa al nivel 0.01 (bilateral).

Fuente: elaboración propia 
Respecto a la evaluación de las diferencias de género se obtuvieron los estadísticos descriptivos del Brief COPE (Tabla 3).

Como se observa en la Tabla 3, las medias por género de las estrategias relacionadas con el afrontamiento centrado en la solución, en el humor y en uso de sustancias son mayores en los varones. Y las medias relacionadas con la desconexión-autoinculpación, el apoyo socioemocional, la religión, la aceptación y la evasión son mayores en las mujeres.
Para distinguir si las diferencias son significativas se utilizó la prueba $t$ de Student para muestras independientes, encontrándose que existen diferencias significativas $(p<.05)$ entre grupos por sexo en las estrategias de apoyo socioemocional, religión, humor y evasión, como se muestra en la Tabla 3.

En cuanto a los factores de personalidad del NEO PI-R (Costa \& McCrae, 1992), los estadísticos de grupo indican medias mayores en las mujeres en cada uno de los Cinco Grandes (Tabla 4); pero sólo

TABLA 3

Estadísticos descriptivos y diferencias por género ( $t$ de Student) para el Brief COPE

\begin{tabular}{|c|c|c|c|c|c|}
\hline & Sexo & Media & D.T. & $t$ & $p$ \\
\hline \multirow{2}{*}{ Afrontamiento centrado en la solución } & Varón & 12.98 & 2.888 & \multirow{2}{*}{-0.279} & \multirow{2}{*}{0.788} \\
\hline & Mujer & 12.93 & 2.887 & & \\
\hline \multirow{2}{*}{ Desconexión-Autoinculpación } & Varón & 3.57 & 2.025 & \multirow{2}{*}{1.079} & \multirow{2}{*}{0.281} \\
\hline & Mujer & 3.72 & 2.044 & & \\
\hline \multirow{2}{*}{ Apoyo Socioemocional } & Varón & 6.36 & 2.768 & \multirow{2}{*}{4.639} & \multirow{2}{*}{0} \\
\hline & Mujer & 7.23 & 2.653 & & \\
\hline \multirow{2}{*}{ Evasión } & Varón & 6.31 & 2.659 & \multirow{2}{*}{6.079} & \multirow{2}{*}{0} \\
\hline & Mujer & 7.47 & 2.845 & & \\
\hline \multirow{2}{*}{ Religión } & Varón & 3.65 & 1.863 & \multirow{2}{*}{3.276} & \multirow{2}{*}{0.001} \\
\hline & Mujer & 4.06 & 1.757 & & \\
\hline \multirow{2}{*}{ Uso de Sustancias } & Varón & 0.29 & 0.799 & \multirow{2}{*}{-1.256} & \multirow{2}{*}{0.209} \\
\hline & Mujer & 0.22 & 0.734 & & \\
\hline \multirow{2}{*}{ Humor } & Varón & 2.75 & 1.476 & \multirow{2}{*}{-1.976} & \multirow{2}{*}{0.049} \\
\hline & Mujer & 2.55 & 1.484 & & \\
\hline \multirow[t]{2}{*}{ Aceptación } & Varón & 3.35 & 1.288 & 217 & 0.828 \\
\hline & Mujer & 3.37 & 1.339 & & \\
\hline
\end{tabular}

Fuente: elaboración propia

TABLA 4

Estadísticos descriptivos por género y valor de t para el NEO PI-R

\begin{tabular}{lccccc}
\hline & Sexo & Media & DE & $t$ & $p$ \\
\hline \multirow{2}{*}{ Neuroticismo } & Varón & 87.37 & 18.182 & 7.342 & 0 \\
\multirow{2}{*}{ Extraversión } & Mujer & 97.04 & 20.2 & & \\
\multirow{2}{*}{ Apertura } & Varón & 120.08 & 19.141 & 1.145 & 0.253 \\
& Mujer & 121.53 & 17.782 & & \\
Amabilidad & Varón & 105.43 & 12.423 & 0.322 & 0.747 \\
Responsabilidad & Mujer & 105.72 & 13.064 & & \\
& Varón & 116.28 & 15.362 & 1.463 & 0.144 \\
& Mujer & 117.78 & 14.236 & 0.043 & 0.965 \\
\hline
\end{tabular}

Fuente: elaboración propia 
se encontró diferencia significativa $(p<0.05)$ por medio de la $t$ de Student en el factor Neuroticismo.

\section{Discusión}

Para el presente estudio, se planteó como primer objetivo evaluar las propiedades psicométricas Brief COPE, respecto a lo cual se afirma que aunque el valor del KMO y la prueba de esfericidad de Barlett evidenciaban la factibilidad del AFE, su validez no es concluyente, porque no se confirma la estructura original de la escala en sus 14 factores (Carver, 1997).

De igual manera, la fiabilidad que se estimó en un primer momento de las 14 subescalas fue baja; la misma que al realizarse el AFE y obtener una nueva dimensionalidad de la escala, ahora con 8 factores, se incrementó logrando categorizarse como alta en el $50 \%$ de estos.

Estadística y teóricamente la estructura obtenida podría ser la mejor estructura del instrumento, ya que las cargas factoriales de cada uno de sus ítems, exceptuando el ítem eliminado, acorde con Hair et al. (1999) son adecuadas (oscilan de 0.463 a 0.823). Así, como la integración de varios factores en sus agrupaciones se considera lógica, como lo son la unión de las escalas de Planificación, Reinterpretación Positiva y Afrontamiento Activo que hablan de una forma correcta y positiva (funcional) de afrontar las situaciones estresantes, igualmente, las escalas de Apoyo Emocional y Apoyo Social hablan de la búsqueda de ayuda. Las escalas de Desahogo, Autodistracción y Negación hacen referencia a la evasión del afrontamiento. Y, por último, las escalas de Autoinculpación y Desconexión se relacionan en que sus ítems son un ataque hacia el self del sujeto.

Asimismo, la estructura descrita ha sido corroborada por el AFC, que después del diseño de tres modelos ha arrojado un modelo de ocho factores correlacionados con índices de ajuste adecuados acorde con los criterios de Schermelleh-Engel et al. (2003), Jöreskog y Sörbom (1984) y Browne y Cudeck (1993).

Respecto al objetivo que versa sobre "Evaluar las diferencias de género en el NEO PI-R y el Brief COPE", se enfatiza el hecho de que en las escalas del Brief COPE en las que puntúa más alto la mujer están relacionadas con necesidades de apoyo social, emocional y espiritual, así como con indicadores de pensamientos y conductas que la llevan a dañarse a sí misma por medio de la culpa, la desconexión y la evasión. Lo cual puede estar influido por el contexto sociocultural que enfrenta la mujer. Y respecto al hombre, sucede algo similar, pero con expectativas contrarias ya que los hombres puntuaron alto en estrategias relacionadas con el afrontamiento centrado en la solución, en el humor y en uso de sustancias. Lo que también tiene relación con las expectativas de la sociedad sobre la forma de responder ante situaciones de estrés del hombre, recalcando que las diferencias significativas se localizaron en apoyo socioemocional, evasión, religión (mayor en mujeres) y humor (mayor en los hombres). Los resultados mencionados son similares a los encontrados recientemente por Canabach, Fariña, Freire, González y Del Mar (2013), quienes ponen de manifiesto que los hombres recurren en mayor medida a las estrategias de reevaluación positiva y planificación como medidas de afrontamiento, mientras que las mujeres optan fundamentalmente por la búsqueda de apoyo.

Respecto a los cinco grandes factores de la personalidad (NEO PI-R), se halló que las mujeres presentan puntuaciones más altas en los cinco factores, pero sólo es significativa esa diferencia en Neuroticismo, ya que probablemente sea el factor más característico en el sexo femenino. Empero, previamente De Miguel (2005) sí encontró diferencias significativas en cuatro de las cinco escalas (exceptuando Responsabilidad).

Los hallazgos de este estudio muestran que las respuestas a la adversidad y al estrés se pueden categorizar de diferentes maneras. Se coincide con otros investigadores (Connor-Smith \& Flachsbart, 2007) en considerar que algunas de estas reacciones resultan ser funcionales (centradas en la solución) como lo son afrontamiento activo, planificación y reinterpretación positiva, otras resultan ser menos comprometidas con la acción y llevan a la renuncia de la búsqueda de soluciones (desconexión-auto-inculpación) o a la evasión (desahogo, distracción y negación) y otras más 
pueden resultar neutras como el humor, el acudir a la religión o la búsqueda de apoyo social por razones emocionales.

También en este estudio queda reflejado cómo los cinco factores de la personalidad están implicados en el afrontamiento enfocado a la solución del problema. Se pone de manifiesto igualmente que el neuroticismo se relaciona positivamente con todas las estrategias consideradas disfuncionales.

Cuando Folkman y Moskowitz (2004) repasaron los avances realizados en la investigación sobre afrontamiento en adultos, señalaron que los desafíos más importantes a los que se tienen que enfrentar los investigadores son la clasificación de las estrategias o funciones del afrontamiento y los problemas relacionados con la medición de la efectividad del afrontamiento.

Como se evidencia en este estudio, la estructura factorial original del instrumento que aquí se evalúa (Brief COPE) no se ha podido confirmar en la población brasileña, tal como en una investigación previa ya lo habían reportado Pais-Ribeiro y Rodrigues (2004). Por lo que como aportación al presente, y para asegurar la validez y confiabilidad del Brief COPE en población brasileña, por medio del back translation se tradujo el instrumento original de Carver (1997); además de realizarse las adecuaciones culturales correspondientes a los ítems que así lo requirieron. Sometiéndose a juicio de expertos dichas modificaciones, dio por resultado el instrumento presentado en el Apéndice.

En consecuencia, se invita a los investigadores interesados en el tema a seguir aportando evidencias sobre la evaluación de las propiedades psicométricas de esta propuesta, en estudios rigurosos de muestras brasileñas representativas.

\section{Referencias}

Barlett, M. S. (1950). Tests of significance in factor analysis. British Journal of Statistical Psychology, 3(2), 77-85.

Barlett, M. S. (1951). A further note on tests of significance in factor analysis. British Journal of Statistical Psychology, 4(1), 1-2.
Bolger, N. (1990). Coping as a personality process: A prospective study. Journal of Personality and Social Psychology, 59(3), 525-537.

Bolger, N., \& Zuckerman, A. (1995). A framework for studying personality in the stress process. Journal of Personality and Social Psychology, 69(5), 890-902.

Browne, M., \& Cudeck, R. (1993). Alternative ways of assessing model fit. En K. A. Bollen \& L. J. Scott (Eds.), Testing structural equation models (pp. 136162). Newbury Park, CA: Sage.

Canabach R., Fariña, F., Freire, C., González, P., \& Del Mar, M. (2013). Diferencias en el afrontamiento del estrés en estudiantes universitarios hombres y mujeres. European Journal of Education and Psychology, 6(1), 19-32.

Carver, C. S. (1997). You want to measure coping but your protocol's too long: Consider the BriefCOPE. International Journal of Behavioral Medicine, 4(1), 92-100.

Carver, C. S., \& Connor-Smith, J. (2010). Personality and coping. Annual Review of Psychology, 61, 679-704.

Carver, C. S., Scheier, M. E., \& Weintraub, J. K. (1989). Assessing coping strategies: A theoretically based approach. Journal of Personality and Social Psychology, 56(2), 267-283.

Connor-Smith, J., \& Flachsbart, C. (2007). Relations between personality and coping: A meta-analysis. Journal of Personality and Social Psychology, 93(6), 1080-1107.

Costa, P. T., \& McCrae, R. R. (1985). The NEO Personality Inventory Manual. Odessa, FL: Psychological Assessment Resources.

Costa, P. T., \& McCrae, R. R. (1992). Revised NEO Personality Inventory (NEO PI-R) and NEO FiveFactor Inventory (NEO FFI) manual. Odessa, FL: Psychological Assessment Resources.

Costa, P. T., McCrae R. R., \& Dye, D. (1991). Facet scales for agreeableness and conscientiousness: A revision of the NEO Personality Inventory. Personality and Individual Differences, 12(9), 887-898.

DeLongis, A., \& Holtzman, S. (2005). Coping in context: The role of stress, social support, and personality in coping. Journal of Personality, 73(6), 1-24.

De Miguel, A. (2005). Diferencias de edad y género en el NEO PI-R en dos muestras con distinto nivel 
académico. International Journal of Psychology and Psychological Therapy, 1(1), 13-31.

Fabrigar, L., MacCallum, R., Wegener, D. T., \& Strahan, E. J. (1999). Evaluating the use of exploratory factor analysis in psychological research. Psychological Methods, 4(3), 272-299.

Folkman, S., \& Lazarus, R. S. (1988). Ways of Coping Questionnaire: Research edition manual. Palo Alto, CA: Consulting Psychologists Press.

Folkman, S., \& Moskowitz, J. T. (2004). Coping: Pitfalls and promise. Annual Review of Psychology, $55,745-774$.

Gunzerath, L., Connelly, B., Albert, P., \& Knebel, A. (2001). Relationship of personality traits and coping strategies to quality of life in patients with alpha-1 antitrypsin deficiency. Psychology, Health \& Medicine, 6(3), 335-341.

Hair, J., Anderson, R., Tatham, R., \& Black, W. (1999). Análisis Multivariante (4.a ed.). Barcelona: Prentice Hall.

Hernández-Sampieri, R., Fernández-Collado, C., \& Baptista-Lucio, P. (2010). Metodología de la investigación (4.a ed.). México: McGraw Hill.

Jöreskog, K., \& Sörbom, D. (1984). LISREL-VI user's guide (3.a ed.). Mooresville, IN: Scientific Software.

Landero, R., \& González, M. (2006). Estadística con SPSS y metodología de la investigación. México: Trillas.

Lazarus, R. S., \& Folkman, S. (1984). Stress, appraisal, and coping. Nueva York: Springer.

Lodi-Smith, J., \& Roberts, B. (2012). Concurrent and prospective relationships between social engagement and personality traits in older adulthood. Psychology and Aging, 27(3), 720-727.

McAdams, D. P., \& Olson, B. D. (2010). Personality development: Continuity and change over the life course. Annual Review of Psychology, 61, 517-542.

McCrae, R. R., \& Costa, P. T. (2008). The Five-Factor Theory of personality. En O. P. John, R. W. Robins \& L. A. Pervin (Eds.), Handbook of personality: Theory and research (3.a ed., pp. 159-181). Nueva York: Guilford.
McCrae, R., \& John, O. P. (1992). An introduction to the Five-Factor Model and its applications. Journal of Personality, 60(2), 175-215.

Morán, C. (2006). El cansancio emocional en servicios humanos. Relación con acoso psicológico, personalidad y afrontamiento. Revista de Psicología del Trabajo y de las Organizaciones, 22(2), 227-239.

Morán, C., Landero, R., \& González, M. T. (2010). COPE-28: un análisis psicométrico de la versión en español del Brief COPE. Universitas Psychologica, 9(2), 543-552.

O'Brien, T. B., \& DeLongis, A. (1996). The interactional context of problem-, emotion-, and relationshipfocused coping: The role of Big Five personality factors. Journal of Personality, 64, 775-813.

Pais-Ribeiro, J. L., \& Rodrigues, A. P. (2004). Questões acerca do coping: A propósito do estudo de adaptação do Brief Cope. Psicologia, Saúde e Doenças, 5(1), 3-15.

Ruiz, M. (2000). Introducción a los modelos de ecuaciones estructurales. Madrid: Ediciones UNED.

Sandín, B. (2003). El estrés: un análisis basado en el papel de los factores sociales. Revista Internacional de Psicología Clínica y de la Salud, 3(1), 141-157.

Shiloh, S., \& Orgler-Shoob, M. (2006). Monitoring: A dual-function coping strategy. Journal of Personality, 74(2), 457-478.

Schermelleh-Engel, K., Moosbrugger, H., \& Müller, H. (2003). Evaluating the fit of structural equation models: Test of significance and descriptive goodness-of-fit measures. Methods of Psychological Research Online, 8(2), 23-74.

Somerfield, M. R., \& McCrae, R. R. (2000). Stress and coping research. Methodological challenges, theoretical advances, and clinical applications. American Psychologist, 55(6), 620-625.

Vollrath, M. (2001). Personality and stress. Scandinavian Journal of Psychology, 42(4), 335-347.

Watson, D., \& Hubbard, B. (1996). Adaptational style and dispositional structure: Coping in the context of the Five-Factor Model. Journal of Personality, 64(4), 737-774. 


\section{Apéndice}

\section{BRIEF COPE}

(Sugerencias para la versión brasileña)

Instruções: As frases abaixo descrevem formas de pensar, sentir ou comportar-se que as pessoas costumamos utilizar para enfrentar os problemas pessoais ou situações difíceis, os quais provocam tensão e estresse. As formas de enfrentar os problemas, como estão descritas aqui, não são nem boas nem más, tão pouco umas são melhores ou piores que outras. Simplesmente, algumas pessoas utilizam mais umas formas que outras. Coloque 0, 1, 2, ou 3 no espaço ao lado, escolhendo o número que melhor reflita sua forma de enfrentar os problemas. Obrigado.

\begin{tabular}{|c|c|c|c|}
0 & 1 & 2 & 3 \\
\hline Não, em absoluto & Um pouco & Bastante & Muito \\
\hline
\end{tabular}

\begin{tabular}{|l|l|}
\hline 1. Tento conseguir que alguém me ajude ou aconselhe sobre o que fazer. & \\
\hline 2. Concentro meus esforços em fazer algo sobre a situação em que estou. & \\
\hline 3. Aceito a realidade diante do ocorrido. & \\
\hline 4. Recorro ao trabalho ou a outras atividades para afastar as coisas da minha mente. & \\
\hline 5. Digo a mim mesmo "isto não é real. & \\
\hline 6. Tento propor uma estratégia sobre o que fazer. & \\
\hline 7. Faço brincadeira (piadas) sobre os problemas. & \\
\hline 8. Critico a mim mesmo. & \\
\hline 9. Consigo apoio emocional dos outros. & \\
\hline 10. Tomo medidas para tentar melhorar a situação. & \\
\hline 11. Desisto de tentar ocupar-me com os problemas. & \\
\hline 12. Digo coisas para extravasar meus sentimentos desagradáveis. & \\
\hline 13. Me nego a crer que tenha acontecido. & \\
\hline 14. Tento ver o problema com outros olhos, para fazer que pareça mais positivo. & \\
\hline 15. Utilizo álcool ou outras drogas para me sentir melhor. & \\
\hline 16. Tento encontrar consolo na religião, na minha religião ou crenças espirituais. & \\
\hline 17. intento consolo e compreensão de alguém. & \\
\hline 18. Procuro algo bom no que está acontecendo. & \\
\hline 19. Rio da situação. & \\
\hline 20. Rezo ou medito. & \\
\hline 21. aprendo a viver com o problema. & \\
\hline 22. Faço algo para pensar menos no problema, tal como ir ao cinema o ver televisão. & \\
\hline 23. Expresso meus sentimentos negativos. & \\
\hline 24. Recorro ao álcool ou outras drogas para ajudar a superar o problema. & \\
\hline 25. Desisto de tentar fazer frente ao problema. & \\
\hline 26. Penso atentamente sobre os passos a seguir. & \\
\hline 27. Jogo a culpa sobre o ocorrido. & \\
\hline 28. Consigo que outras pessoas me ajudem ou me aconselhem. & \\
\hline & \\
\hline
\end{tabular}




\section{Correção: Colocar a pontuação dada o cada item e faz cada soma}

\begin{tabular}{|c|c|c|}
\hline AA. Itens 2 & Pl. Itens $6 \_26$ & AE. Itens 9 \\
\hline AS. Itens $1 \_28$ & Re. Itens $16 \_20$ & RP. Itens 14 \\
\hline Ac. Itens $3 \_21$ & Ne. Itens 5 & Hu. Itens 7 \\
\hline AD. Itens $4 \_22$ & AI. Itens $8 \_27 \_=$ & DC. Itens $11 \_25$ \\
\hline Di. Itens 12 & US. Itens 15 & \\
\hline
\end{tabular}

